Vol. 15(11), pp. 392-400, 16 March, 2016

DOI: 10.5897/AJB2015.14874

Article Number: 2ACC70257539

ISSN 1684-5315

Copyright (C) 2016

African Journal of Biotechnology

Author(s) retain the copyright of this article

http://www.academicjournals.org/AJB

Full Length Research Paper

\title{
Response of African eggplants to Fusarium spp. and identification of sources of resistance
}

\author{
Phoebe Kirigo Mwaniki ${ }^{1^{*}}$, Mathew Musumbale Abang ${ }^{2,5 \#}$, Isabel Nyokabi Wagara ${ }^{3 \#}$, Joseph \\ Ngwela Wolukau ${ }^{1}$ and Schroers Hans-Josef ${ }^{4 \#}$ \\ ${ }^{1}$ Department of Crops, Horticulture and Soil Science, Egerton University, Kenya. \\ ${ }^{2}$ AVRDC-The World Vegetable Center, Arusha, Tanzania. \\ ${ }^{3}$ Department of Biological Sciences, Egerton University, Kenya. \\ ${ }^{4}$ Agricultural Institute of Slovenia, Ljubljana, Slovenia. \\ ${ }^{5}$ International Center for Tropical Agriculture (CIAT), Kampala, Uganda.
}

Received 20 July, 2015; Accepted 5 November, 2015

\begin{abstract}
Eggplant (Solanum spp.) production in Arumeru district and other parts of Africa is severely affected by wilting diseases of unknown etiology. Fusarium spp. characterized through morphological and sequence analysis of the translation elongation factor associated with Fusarium wilt of eggplants was used to test the response of three different eggplant species. Three Solanum spp. accessions were tested in a screen house at the seedling stage for resistance to two isolates each of Fusarium equiseti (corda) Sacc, Fusarium solani (Mart.) Sacc and Fusarium oxysporum (Schlecht). The study indicated that accessions MM 1131 (Solanum macrocapon) and $\mathrm{N} 19$ (Solanum anguivi) accessions are susceptible to $F$. equiseti. Accession N 19 (S. anguivi) was susceptible to $F$. solani while both $N 19$ (S. anguivi) and MM 1131 (S. macrocarpon) was also susceptible to $F$. oxysporum $f$. sp. melongenae. Ninety-three accessions of cultivated and wild eggplants were subsequently evaluated in two screen house trials for resistance to Fusarium wilt. A root dip technique was used to inoculate the accessions with isolate Fs 40 ( $F$. oxysporum f.sp. melongenae). Seventeen of the 93 accessions were found to be resistant and they belonged to Solanum macrocarpon and Solanum aethiopicum species. Accessions in $\mathbf{S}$ melongena were found to be the most susceptible. Eggplant accessions that showed high levels of resistance could potentially serve as valuable sources of Fusarium wilt resistance in eggplant breeding programs in Tanzania and beyond.
\end{abstract}

Key words: African eggplants, Fusarium spp. susceptibility, resistance.

\section{INTRODUCTION}

Eggplant (Solanum spp.) is a multi-species, diploid and seed propagated crop that is cultivated widely in sub- saharan Africa. African eggplant (S. aethiopicum L.) and $S$. macrocarpon $\mathrm{L}$. are the most popular native traditional

*Corresponding author. E-mail: phoebemwan@yahoo.com

\#These authors contributed equally to this work.

Author(s) agree that this article remains permanently open access under the terms of the Creative Commons Attribution License 4.0 International License 
Table 1. Description, source, morphology, molecular identification and pathogenicity of the Fusaria from wilting eggplants (Solanum spp.) that were used in this study.

\begin{tabular}{cllllc}
\hline Isolate code & Host & Site & Collection (2009) & Morphology identification & Differential test \\
\hline FS 3 & S. macrocarpon & WVC & July & F. lacertarum & + \\
FS 22 & S. anguivi & WVC & August & F. solani & + \\
FS 24 & S. melongena & Shangarai & August & F. oxyporum* & + \\
FS 27 & S. aethiopicum gr.Kumba & AVRDC & July & F. equiseti & + \\
FS 35 & S. anguivi & WVC & July & F. solani & + \\
FS 40 & S. melongena & Shangalai & August & F. oxysporum & + \\
\hline
\end{tabular}

Identification followed by an * were isolates confirmed by analysis of the ITS region and the $\alpha$ - elongation factor; + Isolate tested of it's pathogenicity; WVC-ARUSHA: World Vegetable Center-ARUSHA.

vegetables in West, and Central Africa. The African eggplant is widely cultivated as a major source of food and is a rich source of vitamins, fibers and minerals. It is also cultivated for medicinal purposes in some countries of Africa (Shippers, 2002) Losses in eggplant production in Africa due to wilt diseases have not been statistically evaluated. Previous research has shown that Fusarium wilt and Verticillium wilt pathogens are the major causal agents of wilting in eggplants (Kouassi et al., 2014).

The search for sources of resistance to Fusarium wilt pathogens has been done using the wild relatives of $S$. melongena and two genes carrying wilt resistance have been tagged (Mutlu et al., 2008; Toppino et al., 2008). S. anguivi and $S$. aethiopicum have been utilized in breeding programmes for development of disease resistant eggplant varieties (Altinok et al., 2014; Toppino et al., 2008). Several eggplant accessions have also been utilized in the development and production of disease resistant rootstocks for grafting high yielding varieties (Boyaci et al., 2011; Yoshida et al., 2004).

Two eggplant accessions of $S$. aethiopicum gr. Gilo and S. aethiopicum gr. Aculeatum (Solanum integrifolium) are known to carry a gene for resistance designated as Rfo-sa1, to the fungal wilt disease caused by $F$. oxysporum f. sp. melongenae (Toppino et al., 2008). Work done by Altinok et al. (2014) and Iwamoto and Ezura (2006) showed that the high diversity of eggplant germplasm represents a valuable source of wilt resistance genes that could be introgressed into cultivated varieties. The objective of this study was to investigate the differential response of a limited number of African eggplant (Solanum spp.) accessions to a range of Fusarium isolates and to search for sources of resistance to the most virulent isolate.

\section{MATERIALS AND METHODS}

Differential response of African eggplants (Solanum spp.) to inoculation with isolates of Fusarium spp.

Six isolates of Fusarium spp. Coded Fs 24 (1480 JQ244840), Fs 3(1474 JQ244844), Fs 35 (1477 JQ244847), Fs 27(1481 JQ244856), Fs 40(1479 JQ244846), Fs 22 were used in this test. The numbers in parenthesis are accession numbers of the isolates nucleotide sequences deposited in the NCBI genebank. They were isolated from eggplants showing wilting symptoms collected from farmers fields in Arumeru district, AVRDC-RCA eggplant research field (Table 1). Morphological, cultural and molecular characterizations were carried out to confirm the identity of the isolates (Ghoneem et al., 2009; O'Donnell et al., 2009; Seifert, 1996).

Three accessions of Solanum spp., SIVONKWE (S. aethiopicum gr.Gilo), N 19 (S. anguivi), and MM 1131 (S. macrocarpon) were used in this study. Inoculation was done on seedlings at the six leaf stage (Altnolk, 2005). The seedlings were lifted gently from the trays and the soil washed off. The root tip of each seedling was cut to two thirds in length. The roots were immersed for $3 \mathrm{~min}$ in a suspension of $1 \times 10^{6}$ conidia per $\mathrm{ml}$ of $F$. oxysporum $\mathrm{f}$. $\mathrm{sp}$. melongenae harvested from 14 day old cultures grown on PDA at $25^{\circ} \mathrm{C}$. The control plants were inoculated with distilled sterile water. The seedlings were planted in $15 \mathrm{~cm}$ diameter plastic pots containing sterile soil (forest soil mixed with sand at the ratio of 3:1) and placed in a screen house. The experiment was set up in a randomized complete block design with five replicates.

\section{Identification of sources of resistance to eggplant wilt (Fusarium oxysporum f.sp. melongenae)}

One isolate of $F$. oxysporum f.sp. melongenae coded Fs 40 isolated from infected plants of cultivated aubergine ( $S$. melongena) was used. This isolate was used due to its high mean disease index in the differential response test and its high prevalence in the cultivated eggplants (S. melongena).

\section{Preparation of inoculum}

Single spore cultures were grown on PDA, for consistent sporulation and pigmentation, Petri plates were kept $40 \mathrm{~cm}$ below cool white fluorescent tubes and illuminated for $12 \mathrm{~h}$ periods at alternating $25^{\circ} \mathrm{C}$ day $/ 20^{\circ} \mathrm{C}$ night cycles. Conidia was harvested from 14 day old cultures grown on Potato Dextrose Agar (PDA) at $25^{\circ} \mathrm{C}$ by adding sterile water to the plates and scraping the surface of the culture with a sterile glass slide. The resulting conidial suspension was filtered through two layers of cheesecloth to remove mycelia fragments. Spore concentrations were then determined using a hemacytometer and adjusted in distilled water to a concentration of $1 \times 10^{6}$ conidia per $\mathrm{ml}$ which was adopted in the two trials. The inoculum was used to inoculate seedlings in the susceptibility test.

\section{Susceptibility test}

Evaluation for wilt resistance was done according to Yoshiteru et al. 
(1996) with modifications. Ninety three eggplant accessions (Table 2) of S. macrocarpon, S. aethiopicum, S. anguivi, S. dasyphyllum and $S$. melongena were inoculated at 5-week-old stage (2-3 true leaves emerged) using root dip inoculation technique. Six seedlings of each accession were planted in $15 \mathrm{~cm}$ diameter plastic pots containing sterile soil. The soil was drenched with $5 \mathrm{ml}$ of inoculum $\left(1 \times 10^{6}\right.$ spores $\left./ \mathrm{ml}\right)$ of $F$. oxysporum f.sp melongenae and the seedlings grown in a screen house. The experiment was laid out in a randomized complete block design with three replicates.

\section{Disease evaluation and statistical analysis}

\section{Differential response of the eggplants to the isolates}

Plants were monitored daily for wilt development and symptomology and the extent of disease severity recorded at intervals of four weeks starting from the $4^{\text {th }}$ week Scoring was done on a 1 to 5 disease scale where $1=$ no symptoms; 2 = slight wilting and yellowing of the lowest leaves; $3=$ half of the leaves wilted or showing yellowing; 4 = almost all the leaves wilted or showed yellowing; and 5 = all the leaves wilted, showed yellowing or plant died. The response for each accession and cultivar was determined against a mean disease index calculated according to the following formula (Matsubara et al., 2004):

$\sum$ (Number of plants $\times$ degree of symptom)

Total number of plants $\times 5$

\section{Susceptibility test for wilt resistance}

The response for each accession and cultivar was determined against a percentage disease index calculated according to the following formula (Matsubara et al., 2004).

Disease index $=\frac{\sum \text { (number of plants } \times \text { degree of symptom) }}{20}=\times 100$

Total number of plants $\times 5$

Less than $20 \%=$ symptomless; $20-40 \%=$ slight wilting and yellowing of the lowest leaves; $40-60 \%=$ almost all the leaves wilted or showing yellowing; $60-80 \%=$ almost all the leaves wilted or showing yellowing and $80-100 \%$ all the leaves wilted, showing yellowing or plant died. R: Resistant $(<40 \%)$; PR: Partially resistant (40 - 50\%); S: Susceptible (>50).

\section{Statistical analysis}

Data was analyzed for significant differences using ANOVA and comparison of means among accessions was done using Tukeys HSD. Co STAT statistical package (COHORT Software, Minneapolis, USA) was used to analyze the data. The level of probability was set at $P=0.05$. Means on the same column followed by a common letter are not significantly different according to the Tukeys test $(p \leq 0.05)$.

\section{RESULTS}

Differential response of African eggplants (Solanum spp.) to inoculation with isolates of Fusarium spp.

Typical symptoms of eggplant Fusarium wilt were observed when seedlings of MM 1131 and $\mathrm{N} 19$ (susceptible lines) were inoculated with isolates $F$. oxysporum, $F$. solani and $F$. equiseti. Symptoms on the inoculated seedlings included sudden drooping of leaflets starting from the apical part and progressing downward, yellowing of the leaves which began from one side of the leaf and final wilting of the whole plant. These symptom developments were also observed by Beladid et al. (2004). Complete death of the susceptible seedlings occurred during the 5th week.

The differential tests indicated that isolate Fs 27 ( $F$. equiseti) and Fs 24, 40 ( $F$. oxysporum f.sp melongenae) generally resulted to a higher mean disease index (>2.5) across the three accessions used compared to the other isolates which had a disease index of $<2.0$ (Table 3). Fusarium equiseti and $F$. oxysporum were pathogenic to both MM 1131 (S. macrocapon) and N19 (S. anguivi). Solanum anguivi was susceptible to all the Fusarium isolates except $F$. laceratum. Solanum aethiopicum gr. Gilo, (accession Sivonkwe) showed significant resistance to all the isolates except Fs27. Fs 3 was nonpathogenic to any of the Solanum spp (Table 3). Re isolation and culturing of the pathogen from the infected stem tissues on PDA yielded colonies of $F$. oxysporum, $F$. solani and $F$. equiseti inoculated and therefore proved positive for Koch's postulates.

\section{Identification of sources of resistance to eggplant wilt (Fusarium oxysporum f.sp. melongenae)}

The inoculation method adopted gave good disease incidence in all trials, and provided a useful screening system for resistance to Fusarium wilt. Symptoms started after 7 days of inoculation. There were significant differences between the two trials and this was attributed to the different prevailing weather conditions at the time the two trials were carried out. Temperatures were higher and the condition was dry in the duration the $1^{\text {st }}$ trial was carried out compared to the $2^{\text {nd }}$ trial which was characterized by heavy rains and temperatures as low as $13^{\circ} \mathrm{C}$ during the nights. The maximum and minimum temperatures in the $1^{\text {st }}$ trial ranged from 21 to $34^{\circ} \mathrm{C}$ while in the $2^{\text {nd }}$ trial ranged from 13 to $29^{\circ} \mathrm{C}$ therefore the symptoms were more severe. The two trials showed that the strain used in inoculating the accessions (Fs 40) was virulent to the accessions.

$12 \%$ of the accessions tested were considered as resistant while $71 \%$ were partially resistant and $10 \%$ susceptible According to Table 4, MM 1044, MM 11044 and MM 10260 were accessions found to be resistant within the $S$. macrocarpon species. There were also very susceptible accessions found in the $S$. macrocarpon species such as MM 855 and MM 283. This shows a high genetic variability within this species and an assumption on this species being totally susceptible or totally being resistant is ruled out. There were no accessions within 
Table 2. List of eggplant species and cultivars tested.

\begin{tabular}{|c|c|c|}
\hline Species & Cultivar & Origin \\
\hline S. aethiopicum & SOS 1 & AVRDC gene bank ${ }^{*}$ \\
\hline S. aethiopicum & UG-AE-4 & Uganda \\
\hline S. aethiopicum & UG-AE-10 & Uganda \\
\hline S. aethiopicum & UG-AE-21 & Uganda \\
\hline S. aethiopicum & TZSMN 2-8 & Tanzania \\
\hline S. aethiopicum & TZSMN 3-10 & Tanzania \\
\hline S. aethiopicum & TZSMN 75-7 & Tanzania \\
\hline S. aethiopicum & $M L-A E-5$ & Malawi \\
\hline S. aethiopicum & ML-AE-12 & Malawi \\
\hline S. aethiopicum & DB3 & Ghana \\
\hline S. aethiopicum & Manyire Green & Tanzania \\
\hline Solanum spp. & Landrace & Tanzania \\
\hline S. aethiopicum & OAA (089) & Cameroon \\
\hline S. aethiopicum & Small oval & Tanzania \\
\hline S. aethiopicum & N20 & AVRDC gene bank ${ }^{*}$ \\
\hline S. aethiopicum & Heart shaped & Tanzania \\
\hline S. aethiopicum & Sivonkwe & AVRDC gene bank* \\
\hline S. aethiopicum gr. shum & MM347 & Congo \\
\hline S. aethiopicum & MM01150 & AVRDC gene bank* \\
\hline S. aethiopicum gr. Gilo & MM1371 & Tanzania \\
\hline S. aethiopicum & MM1106 & AVRDC gene bank* \\
\hline S. aethiopicum gr. Aculeatum & MM134 & France \\
\hline S. aethiopicum gr. Aculeatum & MM1474 & INDE \\
\hline S. aethiopicum gr. Aculeatum & MM1102 & Burkina Faso \\
\hline S. aethiopicum gr. Aculeatum & MM1483 & Inconnue \\
\hline S. aethiopicum gr. Gilo & MM10181 & Ghana \\
\hline S. aethiopicum gr. Gilo & MM803 & Gabon \\
\hline S. aethiopicum gr. Gilo & MM870 & Madagascar \\
\hline S. aethiopicum gr. Gilo & MM1641 & Africa( Ouest) \\
\hline S. aethiopicum gr. Gilo & MM10086 & Togo \\
\hline S. aethiopicum gr. Gilo & MM10245 & Zambia \\
\hline S. aethiopicum gr. Gilo & MM1480 & Inconnue \\
\hline S. aethiopicum gr. Gilo & MM1188 & Inconnue \\
\hline S. aethiopicum gr. Gilo & MM11010 & Cote D' Voire \\
\hline S. aethiopicum gr. Gilo & MM196 TER & Burkina Faso \\
\hline S. aethiopicum gr. Gilo & MM868 & Tchad ( Bousso) \\
\hline S. aethiopicum gr. Gilo & MM458 & Japon \\
\hline S. aethiopicum gr. Gilo & MM10079 & Togo \\
\hline S. aethiopicum gr. Gilo & MM10213 & Ghana \\
\hline S. aethiopicum gr. Gilo & MM1162 & Uganda \\
\hline S. aethiopicum gr. Kumba & MM574 & Senegal \\
\hline S. aethiopicum gr. Kumba & MM1642 & Africa( Ouest) \\
\hline S. aethiopicum gr. Kumba & MM1107 & Burkina Faso \\
\hline S. aethiopicum gr. Kumba & MM1207 & Mali \\
\hline S. aethiopicum gr. Kumba & MM267 & Mauritania \\
\hline S. aethiopicum gr. Shum & MM1121 & Zambia \\
\hline S. aethiopicum gr. Shum & MM1119 & Togo \\
\hline S. aethiopicum gr. Shum & MM1161 & Bernin \\
\hline S. aethiopicum & UG-AE-1 & Uganda \\
\hline S. aethiopicum & UG-AE-3 & Uganda \\
\hline S. aethiopicum & UG-AE-14 & Uganda \\
\hline
\end{tabular}


Table 2. Contd.

\begin{tabular}{|c|c|c|}
\hline S. aethiopicum & UG-AE-15 & Uganda \\
\hline S. aethiopicum & UG-AE-23 & Uganda \\
\hline S. aethiopicum & UG-AE-24 & Uganda \\
\hline S. melongena & Black beauty & Tanzania \\
\hline S. dasyphyllum & MM1164 & Togo \\
\hline S. dasyphyllum & MM12126 & Uganda \\
\hline S. macrocarpon & MM10256 & Ghana \\
\hline S. macrocarpon & MM714 & Zimbabwe \\
\hline S. macrocarpon & MM10260 & Ghana \\
\hline S. macrocarpon & MM11044 & Cote D' Voire \\
\hline S. macrocarpon & MM132 & France \\
\hline S. macrocarpon & MM1131 & Togo \\
\hline S. macrocarpon & MM 150 & Cote D' Voire \\
\hline S. macrocarpon & MM283 & AVRDC gene bank ${ }^{*}$ \\
\hline S. macrocarpon & MM12209 & Zaire \\
\hline S. macrocarpon & MM252 & Ghana \\
\hline S. macrocarpon & MM855 & Togo \\
\hline S. macrocarpon & UG-AE-6 & Uganda \\
\hline S. macrocarpon & UVPP & Tanzania \\
\hline S. macrocarpon & CR001 & Cameroon \\
\hline S. macrocarpon & MM01139 & AVRDC gene bank ${ }^{*}$ \\
\hline S. macrocarpon & MM01064 & AVRDC gene bank ${ }^{*}$ \\
\hline S. melongena & S. 00677 & AVRDC gene bank ${ }^{*}$ \\
\hline S. melongena & S. 00718 & AVRDC gene bank ${ }^{*}$ \\
\hline S. melongena & S. 00735 & AVRDC gene bank ${ }^{*}$ \\
\hline S. melongena & S. 00811 & AVRDC gene bank ${ }^{*}$ \\
\hline S. melongena & S. 00017 & AVRDC gene bank ${ }^{*}$ \\
\hline S. melongena & S. 0052 & AVRDC gene bank* \\
\hline S. melongena & S. 00204 & AVRDC gene bank* \\
\hline S. melongena & S. 00256 & AVRDC gene bank ${ }^{*}$ \\
\hline S. melongena & S. 00736 & AVRDC gene bank* \\
\hline S. melongena & S. 00567 & AVRDC gene bank ${ }^{*}$ \\
\hline S.melongena & TS00567 & AVRDC gene bank ${ }^{*}$ \\
\hline S.melongena & TS00131 & AVRDC gene bank ${ }^{*}$ \\
\hline Solanum anguivi & N19 & AVRDC gene bank ${ }^{*}$ \\
\hline S. anguivi & MM1103 & Burkina Faso \\
\hline S. anguivi & MM905 & AVRDC gene bank ${ }^{*}$ \\
\hline S. anguivi & MM159 & AVRDC gene bank ${ }^{*}$ \\
\hline Solanum spp. & TZSMN 15-2 & Tanzania \\
\hline Solanum spp. & ML-AE-4 & Malawi \\
\hline Solanum spp. & ML-AE-6 & Malawi \\
\hline Solanum spp. & ML-AE-9 & Malawi \\
\hline
\end{tabular}

*Germplasm without place of origin data.

the $S$. melongena species categorized as being resistant.. Accesions MM 1161, MM 1616, UG AE- 21, MM 1119 and SOS1 in the $S$. aethiopicum species were resistant to the Fusarium isolate used. This species also exhibited a high genetic variability in its resistance to $F$. oxysporum f.sp. melongenae (FOM). Accessions used within the $S$. anguivi and $S$. dasyphyllum species were found to range from partially resistant to susceptible

\section{DISCUSSION}

The survival and activity of Fusarium spp. is greatly dependant on many factors, with the most important ones being soil moisture, soil and air temperatures (Mui-Yun, 
Table 3. Behavior of three African eggplants after inoculation with different isolates of Fusarium spp.

\begin{tabular}{|c|c|c|c|c|c|c|c|}
\hline \multirow[b]{2}{*}{$\begin{array}{l}\text { Accession } \\
\text { code }\end{array}$} & \multicolumn{7}{|c|}{ Mean disease severity $(1-5) ;$ Isolates tested for pathogenicity } \\
\hline & $\begin{array}{l}\text { Fs } 24 \\
\text { F.oxy }\end{array}$ & $\begin{array}{l}\text { Fs } 35 \\
\text { F.sol } \\
\end{array}$ & $\begin{array}{l}\text { Fs } 22 \\
\text { F.sol } \\
\end{array}$ & $\begin{array}{l}\text { Fs } 27 \\
\text { F. equi }\end{array}$ & $\begin{array}{l}\text { Fs } 3 \\
\text { F. lac }\end{array}$ & $\begin{array}{l}\text { Fs } 40 \\
\text { F.oxy }\end{array}$ & Control \\
\hline MM 1131 & $3^{\mathrm{ab}}$ & $1.2^{\mathrm{cd}}$ & $1.2^{\mathrm{cd}}$ & $3^{\mathrm{ab}}$ & $1.0^{\mathrm{d}}$ & $2.6^{\mathrm{abcd}}$ & $1.2^{\mathrm{cd}}$ \\
\hline N 19 & $2.8^{\mathrm{abc}}$ & $2.6^{\mathrm{abcd}}$ & $2.8^{\mathrm{abc}}$ & $4^{\mathrm{a}}$ & $1.6^{\mathrm{bcd}}$ & $2.8^{\mathrm{abc}}$ & $1.4^{\mathrm{bcd}}$ \\
\hline SIVONKWE & $1.2^{\mathrm{cd}}$ & $1.8^{\mathrm{bcd}}$ & $1.4^{\mathrm{bcd}}$ & $2.4^{\mathrm{abcd}}$ & $1.4^{\mathrm{bcd}}$ & $1.4^{\mathrm{bcd}}$ & $1.0^{\mathrm{d}}$ \\
\hline
\end{tabular}

*Values on each column followed by a letter in common are not significantly different at $(P \leq 0.05)$. Foliar symptom scale (1-5), higher numbers indicate severerity of disease.

Table 4. Reaction of eggplant accessions and cultivars after artificial inoculation with Fusarium oxysporum f.sp melongenae (FS 40) expressed as disease incidence (\%) in the two trials.

\begin{tabular}{|c|c|c|c|c|c|}
\hline Species & Accession code & Trial 1 & Trial 2 & Average & Tukeys test \\
\hline S. macrocarpon & MM 10260 & 36.7 & 20 & $28.3^{R}$ & $\mathrm{e}$ \\
\hline S. aethiopicum & MM 1119 & 33.3 & 23.3 & $28.3^{R}$ & e \\
\hline Solanum spp. & UG AE 6 & 33.3 & 26.7 & $30^{R}$ & e \\
\hline S. aethiopicum & SOS1 & 30 & 33.3 & $31.7^{\mathrm{R}}$ & e \\
\hline S. aethiopicum & MM 1616 & 43.3 & 20 & $31.7^{\mathrm{R}}$ & e \\
\hline Solanum spp. & ML AE 6 & 40 & 26.7 & $33.3^{R}$ & e \\
\hline Solanum spp. & ML AE 4 & 30 & 40 & $35^{\mathrm{R}}$ & de \\
\hline S. aethiopicum & MM 1161 & 36.7 & 36.7 & $36.7^{\mathrm{R}}$ & de \\
\hline S. macrocarpon & MM 11044 & 36.7 & 36.7 & $36.7^{\mathrm{R}}$ & de \\
\hline S. aethiopicum & MM 10079 & 40 & 33.3 & $36.7^{R}$ & de \\
\hline S. aethiopicum & MM 1207 & 40 & 33.3 & $36.7^{\mathrm{R}}$ & de \\
\hline S. aethiopicum & UG AE-21 & 30 & 43.3 & $36.7^{\mathrm{R}}$ & de \\
\hline Solanum spp. & MM 1692 & 40 & 36.7 & $38.3^{R}$ & de \\
\hline S. aethiopicum & TZ SMN AE 3-10 & 50 & 30 & $40^{R}$ & de \\
\hline S. aethiopicum & MM 11008 & 36.7 & 43.3 & $40^{R}$ & de \\
\hline S. macrocarpon & MM 1044 & 46.7 & 33.3 & $40^{R}$ & de \\
\hline Solanum spp. & LANDRACE & 50 & 30 & $40^{\mathrm{R}}$ & de \\
\hline Solanum spp. & MM 01139 & 36.7 & 46.7 & $41.7^{\mathrm{PR}}$ & de \\
\hline S. aethiopicum & MM 1160 & 43.3 & 40 & $41.7^{\mathrm{PR}}$ & de \\
\hline S. aethiopicum & N 20 & 40 & 43.3 & $41.7^{\mathrm{PR}}$ & de \\
\hline Solanum spp. & ML AE 9 GKK 149 & 43.3 & 40 & $41.7^{\mathrm{PR}}$ & de \\
\hline Solanum spp. & MM 1498 & 50 & 33.3 & $41.7^{\mathrm{PR}}$ & de \\
\hline Solanum spp. & SITE 101 & 40 & 43.3 & $41.7^{\mathrm{PR}}$ & de \\
\hline S. aethiopicum & MM 11010 & 50 & 36.7 & $43.3^{P R}$ & de \\
\hline S. aethiopicum & MM 348 & 50 & 36.7 & $43.3^{\mathrm{PR}}$ & de \\
\hline S. dasyphyllum & MM 12126 & 46.7 & 40 & $43.3^{P R}$ & de \\
\hline S. melongena & S 00813 & 46.7 & 40 & $43.3^{P R}$ & de \\
\hline Solanum spp. & UG $A E-23$ & 40 & 46.7 & $43.3^{\mathrm{PR}}$ & de \\
\hline S. aethiopicum & MM 347 & 43.3 & 43.3 & $43.3^{P R}$ & de \\
\hline S. dasyphyllum & MM 1164 & 43.3 & 43.3 & $43.3^{\mathrm{PR}}$ & de \\
\hline S. macrocarpon & MM 1062 & 43.3 & 43.3 & $43.3^{P R}$ & de \\
\hline S. aethiopicum & MM 10086 & 40 & 50 & $45^{\mathrm{PR}}$ & de \\
\hline S. aethiopicum & MM 1106 & 43.3 & 46.7 & $45^{\mathrm{PR}}$ & de \\
\hline S. aethiopicum & MM 1107 & 46.7 & 43.3 & $45^{\mathrm{PR}}$ & de \\
\hline S. aethiopicum & MM 1121 & 46.7 & 43.3 & $45^{\mathrm{PR}}$ & de \\
\hline S. aethiopicum & MM 1162 & 50 & 40 & $45^{\mathrm{PR}}$ & de \\
\hline S. aethiopicum & MM 1642 & 43.3 & 46.7 & $45^{P R}$ & de \\
\hline
\end{tabular}


Table 4. Contd.

\begin{tabular}{|c|c|c|c|c|c|}
\hline S. aethiopicum & MM 803 & 46.7 & 43.3 & $45^{\mathrm{PR}}$ & de \\
\hline S. aethiopicum & SMALL OVAL TYPE & 50 & 40 & $45^{\mathrm{PR}}$ & cde \\
\hline S. aethiopicum & TZ SMN-AE 2-8 & 56.7 & 33.3 & $45^{\mathrm{PR}}$ & cde \\
\hline S. aethiopicum & TZ SMN AE 52-3 & 50 & 40 & $45^{\mathrm{PR}}$ & cde \\
\hline S. aethiopicum & MM 10252 & 46.7 & 43.3 & $45^{P R}$ & cde \\
\hline S. macrocarpon & MM 1048 & 50 & 40 & $45^{\mathrm{PR}}$ & cde \\
\hline S. macrocarpon & MM 12209 & 40 & 50 & $45^{\mathrm{PR}}$ & cde \\
\hline S. macrocarpon & MM 132 & 36.7 & 53.3 & $45^{\mathrm{PR}}$ & cde \\
\hline Solanum spp. & MM 1007 & 40 & 50 & $45^{\mathrm{PR}}$ & cde \\
\hline S. aethiopicum & MM 10213 & 46.7 & 46.7 & $46.7^{\mathrm{PR}}$ & cde \\
\hline Solanum spp. & ML AE 5 29-7 & 46.7 & 46.7 & $46.7^{\mathrm{PR}}$ & cde \\
\hline S. aethiopicum & MM 1102 & 50 & 43.3 & $46.7^{\mathrm{PR}}$ & cde \\
\hline S. aethiopicum & MM 870 & 50 & 43.3 & $46.7^{\mathrm{PR}}$ & cde \\
\hline S. anguivi & MM 159 & 50 & 43.3 & $46.7^{\mathrm{PR}}$ & cde \\
\hline S. melongena & S 00736 & 50 & 43.3 & $46.7^{\mathrm{PR}}$ & cde \\
\hline Solanum spp. & UG AE- 5 & 43.3 & 50 & $46.7^{\mathrm{PR}}$ & cde \\
\hline Solanum spp. & UG $A E-14$ & 43.3 & 50 & $46.7^{\mathrm{PR}}$ & cde \\
\hline S. aethiopicum & MM 574 & 50 & 46.7 & $48.3^{P R}$ & cde \\
\hline S. melongena & 00017 & 43.3 & 53.3 & $48.3^{P R}$ & cde \\
\hline S. melongena & S 00718 & 43.3 & 53.3 & $48.3^{P R}$ & cde \\
\hline S. melongena & TZ 00567 & 30 & 66.7 & $48.3^{P R}$ & cde \\
\hline Solanum spp. & UG AE - 24 & 43.3 & 53.3 & $48.3^{P R}$ & cde \\
\hline S. aethiopicum & MANYIRE GREEN & 53.3 & 46.7 & $50^{P R}$ & bcde \\
\hline S. aethiopicum & MM 10245 & 60 & 40 & $50^{\mathrm{PR}}$ & bcde \\
\hline S. aethiopicum & UG $A E-10$ & 60 & 40 & $50^{\mathrm{PR}}$ & bcde \\
\hline S. aethiopicum & MM 196 & 46.7 & 56.7 & $51.7^{\mathrm{S}}$ & bcde \\
\hline S. melongena & TS 00131 & 56.7 & 46.7 & $51.7^{\mathrm{S}}$ & bcde \\
\hline Solanum spp. & MM 138 & 56.7 & 46.7 & $51.7^{\mathrm{S}}$ & bcde \\
\hline S. aethiopicum & MM 1158 & 60 & 46.7 & $53.3^{\mathrm{S}}$ & bcde \\
\hline S. melongena & OO 204 & 46.7 & 60 & $53.3^{\mathrm{S}}$ & bcde \\
\hline S. aethiopicum & MM 868 & 53.3 & 53.3 & $53.3^{\mathrm{S}}$ & bcde \\
\hline S. aethiopicum & MM 1188 & 56.7 & 53.3 & $55^{\mathrm{s}}$ & bcde \\
\hline S. aethiopicum & MM 1483 & 60 & 50 & $55^{\mathrm{s}}$ & bcde \\
\hline S. aethiopicum & MM 1615 & 50 & 60 & $55^{S}$ & bcde \\
\hline S. melongena & OO 677 & 50 & 60 & $55^{\mathrm{S}}$ & bcde \\
\hline S. aethiopicum & MM 1371 & 56.7 & 56.7 & $56.7^{\mathrm{S}}$ & abcde \\
\hline S. anguivi & MM 905 & 56.7 & 56.7 & $56.7^{\mathrm{S}}$ & abcde \\
\hline S. macrocarpon & MM 150 & 56.7 & 56.7 & $56.7^{\mathrm{S}}$ & abcde \\
\hline S. aethiopicum & MM 134 & 63.3 & 50 & $56.7^{\mathrm{S}}$ & abcde \\
\hline S. aethiopicum & MM 1480 & 66.7 & 46.7 & $56.7^{\mathrm{S}}$ & abcde \\
\hline S. aethiopicum & TZ SMN AE 75-7 & 63.3 & 50 & $56.7^{\mathrm{S}}$ & abcde \\
\hline S. melongena & O० 567 & 60 & 53.3 & $56.7^{\mathrm{S}}$ & abcde \\
\hline S. melongena & S 00735 & 60 & 53.3 & $56.7^{\mathrm{S}}$ & abcde \\
\hline S. aethiopicum & MM 1474 & 56.7 & 60 & $58.3^{\mathrm{S}}$ & abcde \\
\hline S. aethiopicum & MM 267 & 46.7 & 70 & $58.3^{\mathrm{S}}$ & abcde \\
\hline S. macrocarpon & CR 001 & 56.7 & 60 & $58.3^{\mathrm{S}}$ & abcde \\
\hline S. aethiopicum & MM 10181 & 63.3 & 56.7 & $60^{s}$ & abcde \\
\hline S. aethiopicum & DB 3 & 63.3 & 56.7 & $60^{S}$ & abcde \\
\hline S. anguivi & MM 1103 & 60 & 60 & $60^{S}$ & abcde \\
\hline S. melongena & BLACK BEAUTY & 60 & 60 & $60^{\mathrm{s}}$ & abcde \\
\hline S. anguivi & N 19 & 66.7 & 63.3 & $65^{\mathrm{S}}$ & abcd \\
\hline S. macrocarpon & MM 1131 & 63.3 & 66.7 & $65^{S}$ & abcd \\
\hline
\end{tabular}


Table 4. Contd.

\begin{tabular}{llcccc}
\hline S. aethiopicum & MM 1308 & 66.7 & 70 & $68.3^{\mathrm{S}}$ & $\mathrm{ab}$ \\
S. macrocarpon & MM 283 & 70 & 76.7 & $73.3^{\mathrm{S}}$ & $\mathrm{ab}$ \\
S. melongena & OO 256 & 80 & 73.3 & $76.7^{\mathrm{S}}$ & $\mathrm{a}$ \\
S. macrocarpon & MM 855 & 80 & 83.3 & $58.7^{\mathrm{S}}$ & $\mathrm{a}$ \\
\hline
\end{tabular}

Each value is the mean \% disease incidence of six plants. Means followed by the same letter are not significantly different following Tukeys test, $(P \leq 0.05)$.

2003). The significant differences within the two trials were as a result of the prevailing environmental conditions during the time the two trials were carried out. $F$. oxysporum is a warm weather pathogen and wilting is more prevalent when the temperatures are high $\left(28^{\circ} \mathrm{C}\right)$ and in moisture stressed soils. Infected plants may remain symptomless in wet seasons (Lester et al., 1988). This explains the higher disease severity of the accessions in the first trial compared to the second trial. Similar reports indicate that Fusarium spp. requires soil and air temperatures of 25 to $28^{\circ} \mathrm{C}$ (Mui-yun, 2003) to effectively infect their hosts.

Among the isolates used for the pathogenicity test, $F$. equiseti caused the highest wilting in $S$. macrocarpon and $S$. anguivi accessions followed by $F$. oxysporum f.sp melongenae. Pathogenicity of $F$. oxysporum f.sp melongenae to eggplants has also been reported by several authors (Altinok, 2005; Zhuang, 2005; Cho and Shin, 2004), however accession SIVONKWE in the $S$. aethiopicum gr. Gilo proved to be resistant in this case. This study confirms previous work showing accessions of $S$. aethiopicum gr. Gilo to carry resistance to Fusarium oxysporum f.sp melongenae though other accessions within the species have also been found to be susceptible (Stravato and Capelli, 2000; Toppino et al., 2008). The resistant Sivonkwe accession can be used in breeding for resistance to Fusarium spp. and other wilt related pathogens. Fusarium equiseti pathogenicity in eggplants has not been reported and further studies on its economic importance on cultivated eggplants would contribute significantly to wilt control. Fusarium equiseti and $F$. solani have been reported to cause heavy wilting and severe seedling root rot in sunflower (Sharfun-nahar and Mushtaq, 2007). The colonization and pathogenicity of $F$. equiseti on tomatoes has also been observed by Jamiołkowska, (2009). It's pathogenicity has been linked to a pathogenic factor known as equisetin and trichothecenes (Hestbjerg et al., 2002; Wheeler et al., 1999).

The screening of the 93 accesions to F. oxysporum f.sp melongenae exhibited a whole range of reactions, that is, resistance, partial resistance and susceptible. Accessions within the Solanum dasyphyllum species (MM 1164, MM 12126) found to be partially resistant to $F$. oxysporum f.sp melongenae are wild eggplant species which have not been cultivated but these accessions would be valuable if used as rootstocks. S. macrocarpon is not susceptible to most diseases and is resistant to damping off caused by Thielaviopsis basicola (Shippers, 2002). Certain cultivars of S. macrocarpon have been reported to be resistant to Fusarium wilt (Grubben and Denton, 2004). Interspecific hybridization between $S$. macrocarpon and $S$. melongena is known to produce fertile hybrids and therefore can be used in transfer of the resistant traits to cultivated eggplants. Solanum macrocarpon and $S$. dasyphyllum can also be crossed easily and therefore produce fully fertile hybrids (Shippers, 2002). Solanum aethiopicum gr. Gilo accessions exhibited reactions ranging from resistance, partial resistance to susceptibile. Similar results were reported by Stravato and Cappelli (2000). This may be explained by the existence of genetic variability within the $S$. aethiopicum groups Gilo and Shum as reported by Sekara et al. (2007). Solanum aethiopicum groups, Shum and Kumba were found to carry a higher resistance than Group Gilo. Solanum aethiopicum (MM 1161, MM 1616, UG AE- 21, UG AE-6, SOS1, MM 10079, MM 1207 and MM 11008 and TZSM NAE-3-10) are valuable for breeding for resistance. Further evaluation for resistance to Verticillium and bacterial wilts would be important for eggplant improvement. Accession SOS1 (S. aethiopicum gr. Gilo X S. aethiopicum gr. Aculeatum) which was found to be resistant by Toppino et al. (2008) was also resistant in this study. Previous work has reported eggplant ( $S$. melongena) to be susceptible to $F$. solani resulting to crown rot, vascular discoloration and wilt (Nabi et al., 2013; Romberg and Davis, 2007; Chakraborty et al., 2008). Consideration of the Fusarium spp. causing wilt in $S$. anguivi and $S$. macrocarpon is important when breeding for resistance to wilt for accessions within this species. More screening should also be done to test the resistance of the $S$. aethiopicum species groups to $F$. equiseti and $F$. solani.

Categorizing host reactions (resistant, partially resistant, susceptible) can be useful in indicating an accessions response to disease in disease favourable environments. The present study clearly shows that resistance to fusarium wilt exist in non-commercial eggplant germplasm which can be exploited to reduce losses.

\section{Conflict of interests}

The authors have not declared any conflict of interest. 


\section{ACKNOWLEDGEMENTS}

The authors wish to acknowledge Egerton University for hosting the student for the Msc programme and the World vegetable Center-Arusha (formerly AVRDC) who funded this project for study. They also appreciate the director of world vegetable centre for giving the student the opportunity to use the facilities and resources in the organization.

\section{REFERENCES}

Altınok HH (2005). First report of Fusarium wilt of eggplant caused by Fusarium oxysporum f. sp. melongenae in Turkey. Plant Pathol. J. 54:577.

Altinok HH, Fliz CH, Topcu BV (2014). Genetic variability among breeding lines and cultivars of eggplants against Fusarium oxysporum f.sp melongenae from turkey. Phytoparasitica 42:75-84.

Beladid L, Baum M, Fortas Z, Bouznad Z, Eujayl I (2004). Pathogenic and genetic characterization of Algerian isolates of Fusarium oxysporum f. sp. lentis by RAPD and AFLP analysis. Afr. J. Biotechnol. 3:25-31.

Boyaci HF, Unlu A, Abak K (2011). Genetic analysis of resistance to wilt caused by Fusarium (Fusarium oxysporum melongenae) in eggplant (Solanum melongena). Indian J. Agric. Res. 81:812-815.

Chakraborty MR, Chatterjee NC, Quimio TH (2008). Integrated management of fusarial wilt of eggplant (Solanum melongena) with soil solarization. Micol. Aplicada Int. 21:25-36

Cho WD, Shin HD Eds. (2004). List of plant diseases in Korea. Fourth edition, Korean Soc. Plant Pathol. 779 p.

Ghoneem MK, Saber IA, Elwakil MA (2009). Alkaline Seed-Bed: An Innovative Technique for Manifesting Verticillium dahliae on Fennel Seeds. Plant Pathol. J. 8:22-26.

Grubben GJH, Denton OA (2004). Plant Resources of Tropical Africa Vegetables 2. PROTA Foundation, Wagenigen, Netherlands/Backhuys Publishers, Leiden, Netherlands /CTA, Wagenigen, Netherlands. $668 \mathrm{p}$.

Hestbjerg H, Nielsen KF, Thrane U, Elmholt S (2002). Production of trichothecenes and other secondary metabolites by Fusarium culmorum and Fusarium equiseti on common laboratory media and soil organic matter agar: an ecological interpretation. J. Agric. Food Chem. 50:7593-7599.

Iwamoto Y, Ezura H (2006). Efficient plant regeneration from protoplasts of eggplant rootstock cultivar and its wild relatives. Plant Biotechnol. J. 23:525-529.

Jamiołkowska A (2009). Fungi colonizing and leaves of hot pepper plants (Capsicum annum L.) cultivated in field. EJPAU 12:1505-0297.

Kouassi A, Beli-sika E, Tian-bi TN, Alla-N'Nan O, Kouassi AB, N'zi JC, N'Guetta ASP, Toure BT (2014) Identification of three distinct eggplants subgroups within the Solanum aethiopicum Gilo group from Cote d'ivore by morph-agronomic characterization. Agriculture $4: 260-273$

Lester WB, Craig L, Brett AS (1988). Laboratory manual for Fusarium research: incorporating a key and descriptions of common species found in Australasia. University of Sydney Press, $2^{\text {nd }}$ edition. $156 \mathrm{p}$.
Matsubara T, Hirano I, Sassa D, Koshikawa K (2004). Increased tolerance to Fusarium wilt in mycorrhizal strawberry plants raised by capillary watering methods. Environ. Control Biol. 42:185-191.

Mui-Yun W (2003). Fusarium oxysporum f.sp lycopersici (Sacc.) Snyder and Hans. 728. Soilborne plant pathogen class project. NC State University.

Mutlu N, Boyaci FH, Gocmen M, Abak K (2008). Development of SRAP, SRAP-RGA, RAPD and SCAR markers linked with a Fusarium wilt resistance gene in eggplant. Theor. Appl. Genet. 117:1303-1312.

Nabi G, Samrah B, Syed T, Hague E, Athar M, Sultana V, Ara J (2013). Management of root diseases of eggplant and watermelon with application of asafetida and seaweeds. Appl. Bot. Food Qual. J. 86:138-142

O'Donnell K, Sutton DA, Rinaldi MG, Gueidan C, Crous PW, Geiser DM (2009). Novel multilocus sequence typing scheme reveals high genetic diversity of Human pathogenic members of the Fusarium incarnatum- $F$. equiseti and $F$. chlamydosporum species complexes within the United states. J. Clin. Microbiol. 47:3851-3861.

Romberg MK, Davis RM (2007). Host range and phylogeny of Fusarium solani F. sp.eumartii from potato and tomato in California. The American Phytopathological Society. Plant Dis 91:585-592.

Seifert K (1996). FUSKEY Fusarium Interactive key. Agriculture and Agri- Food Canada Product Development Unit, Now taxonomic Information Systems. $65 \mathrm{p}$.

Sękara A, Cebula S, Kunicki E (2007). Cultivated eggplants - origin, breeding objectives and genetic resources, a review. Foliar Hortic. 9:97-114

Sharfun-nahar, Mushtaq M (2007). Pathogenic effects and transmission studies of seed-borne Fusarium species in sunflower. Pak. J. Bot. 39:645-649.

Shippers RR (2002). African indigenous vegetables, An overview of the cultivated species 2002- Revised version on CD-ROM. Natural Resources International Limited, Aylesford, UK.

Stravato VM, Cappelli C (2000). Behaviour of Solanum spp. on inoculation with different isolates of Fusarium oxysporum f. $\mathrm{sp}$. melongenae. Bulletin OEPPEPPO 30:247-249.

Toppino L, Giampiero V, Rotino GL (2008). Inheritance of Fusarium wilt resistance introgressed from Solanum aethiopicum Gilo and Aculeatum groups into cultivated eggplant ( $S$. melongena) and development of associated PCR-based markers. Mol. Breed. 22:237-250.

Wheeler MH, Stipanovic RD, Puckhaber LS (1999). Phytotoxicity of equisetin and epi-equisetin isolated from Fusarium equiseti and $F$. pallidoroseum. Mycol. Res. 103:967-973.

Yoshida T, Monma S, Matsunaga H, Sakata Y, Saito T (2004). Development of a new rootstock eggplant cultivar 'Daizaburou' with high resistance to bacterial wilt and Fusarium wilt. Yasai Chagyo Kenkyujo Kenkyu Hokoku 3:199-211.

Yoshiteru S, Monma S, Tomoaki N, Komochi S (1996). Evaluation of resistance to bacterial wilt and Verticillium wilt in Eggplants (Solanum melongena L.) collected in Malaysia. J. Jpn. Soc. Hortic. Sci. J. 65: 81-88.

Zhuang Y (2005). Fungi of northwestren China. Mycotaxon, Ltd., Ithaca, NY, $430 \mathrm{p}$. 